Article

\title{
Novel Method for Virtual Restoration of Cultural Relics with Complex Geometric Structure Based on Multiscale Spatial Geometry
}

\author{
Miaole Hou ${ }^{1,2}$, Su Yang ${ }^{3, *}$, Yungang $\mathrm{Hu}^{1,2, *}$, Yuhua $\mathrm{Wu}^{4}$, Lili Jiang ${ }^{5}$, Sizhong Zhao ${ }^{1,2}$ \\ and Putong Wei ${ }^{1,2}$ \\ 1 School of Geomatics and Urban Information, Beijing University of Civil Engineering and Architecture, \\ Beijing 100044, China; houmiaole@bucea.edu.cn (M.H.); 210813j116013@stu.bucea.edu.cn (S.Z.); \\ 210813j117014@stu.bucea.edu.cn (P.W.) \\ 2 Beijing Key Laboratory for Architectural Heritage Fine Reconstruction \& Health Monitoring, \\ Beijing University of Civil Engineering and Architecture, Beijing 102616, China \\ 3 North China Institute of Computing Technology, Beijing 100083, China \\ 4 Historic Cultural Research Institute, Beijing 100029, China; wuyuhua@cahch.org.cn \\ 5 Beijing Digsur Science and Technology Co. Ltd., Beijing 100012, China; jiangll18810970196@outlook.com \\ * Correspondence: cinder0711@hotmail.com (S.Y.); huyungang@bucea.edu.cn (Y.H.); \\ Tel.: +86-138-1100-7050 (S.Y.); +86-136-4134-0555 (Y.H.)
}

Received: 18 July 2018; Accepted: 9 August 2018; Published: 27 August 2018

\begin{abstract}
Because of the age of relics and the lack of historical data, the geometric forms of missing parts can only be judged by the subjective experience of repair personnel, which leads to varying restoration effects when the geometric structure of the complex relic is reconstructed. Therefore, virtual repair effects cannot fully reflect the historical appearance of cultural relics. In order to solve this problem, this paper presents a virtual restoration method based on the multiscale spatial geometric features of cultural relics in the case of complex construction where the geometric shape of the damaged area is unknown, using the Dazu Thousand-Hand Bodhisattva statue in China as an example. In this study, the global geometric features of the three-dimensional (3D) model are analyzed in space to determine the geometric shape of the damaged parts of cultural relics. The local geometric features are represented by skeleton lines based on regression analysis, and a geometric size prediction model of the defective parts is established, which is used to calculate the geometric dimensions of the missing parts. Finally, 3D surface reconstruction technology is used to quantitate virtual restoration of the defective parts. This method not only provides a new idea for the virtual restoration of artifacts with complex geometric structure, but also may play a vital role in the protection of cultural relics.
\end{abstract}

Keywords: cultural heritage; virtual restoration; spatial geometric features; skeleton line; regression analysis; Dazu Thousand-Hand Bodhisattva statue

\section{Introduction}

Computer-aided restoration of cultural heritage relics has made substantial progress, yet it still faces major challenges when the geometry of the damaged parts of artifacts is unknown. Due to the lack of reliable evidence for restoration, the geometry of the missing parts of many artifacts cannot be specified. As a result, for the same piece of an artifact, different persons may repair its incomplete parts differently, and its true historical appearance cannot be determined. As shown in Figure 1, in a part of the Dazu Thousand-Hand Bodhisattva statue, due to its long history and erosion, the index finger is missing. In order to provide a reference for the actual repair of this finger, a computer may be used 
to perform virtual restoration. However, due to a lack of reliable evidence for the virtual restoration, different restoration methods can lead to different repair results (see Figure 1b-d).

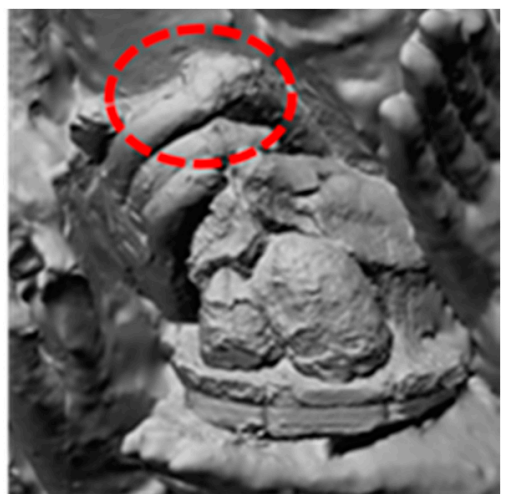

(a)

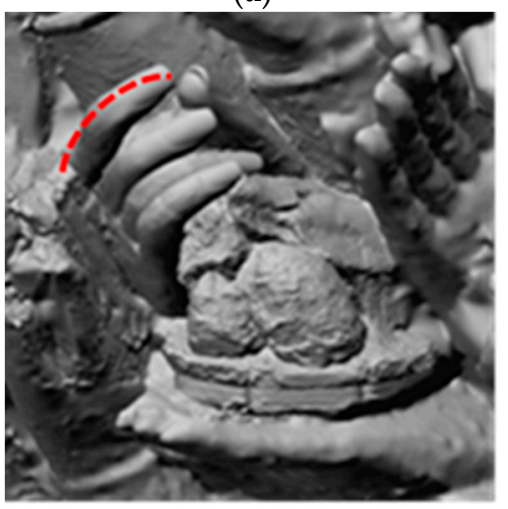

(c)

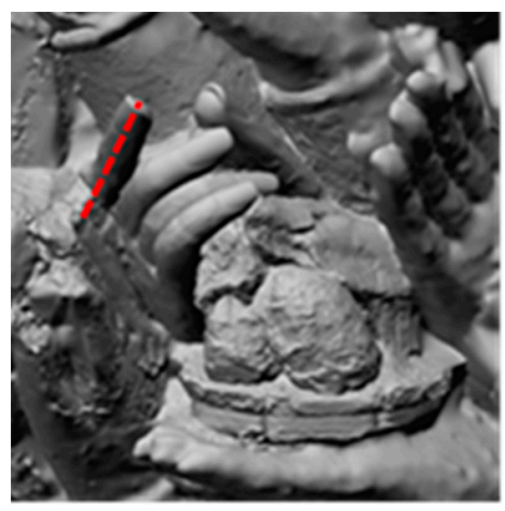

(b)

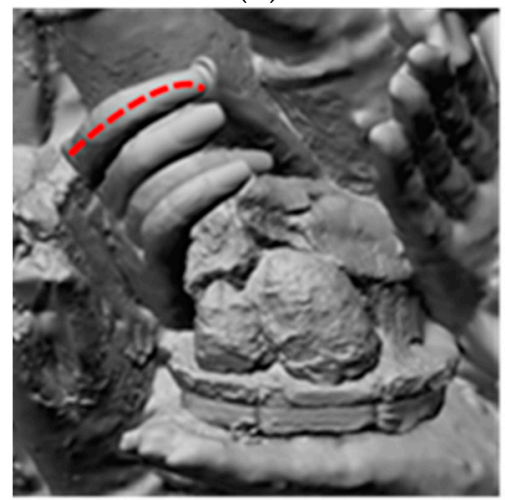

(d)

Figure 1. Different restoration results due to lack of reliable restoration evidence: (a) damaged finger; (b) possible result 1 ; (c) possible result 2; and (d) possible result 3.

Therefore, for damaged and missing parts of an artifact, it is essential to find evidence for virtual restoration by analyzing its basic geometry in the same period or of the same type, so that the effect of virtual restoration can be close to its true historical appearance. This not only helps with the three-dimensional display of artifacts, but also provides a more accurate repair plan for artifact protection, so that valuable historical artifacts can be continuously preserved. Hence, this problem has become an important and timely issue that needs to be solved in the field of virtual artifact restoration.

In response to these problems, this paper presents a virtual restoration method for artifacts with complex geometric structure based on multiscale spatial geometric features, by using virtual restoration of defective fingers in the Dazu Thousand-Hand Bodhisattva statue as an example. In this study, the basic geometric features of the missing parts are determined by analyzing the global geometric features, such as the symmetry and similarity of the three-dimensional models. High-precision three-dimensional models of cultural relics are extracted from the skeleton lines as the local geometric features of the relics, and the geometric dimensions of the missing parts are determined by mathematical analysis.

The paper is organized as follows. After this introduction, some related works are reviewed in Section 2, and Section 3 introduces the overall process of the proposed method and the key methods in the process. Section 4 describes the experimental application of the proposed method using the Dazu Thousand-Hand Bodhisattva statue. Section 5 provides some discussion about the method. Section 6 concludes the paper with observations obtained from this study. 


\section{Related Works}

In the field of virtual artifact restoration, some progress has already been made with regard to the technologies used for virtual restoration of broken artifacts. For example, Su [1] used historical records, pictures, and an analysis of the style of the Qingzhou Buddha statues to restore one of the statues by computer. However, due to their long history, no reliable information is available for many artifacts. Many researchers [2-4] have investigated techniques for extraction and matching of surface boundaries of artifact fragments to provide more reliable repair information. For example, Fan [5] applied shape matching of complementary three-dimensional (3D) polygonal arcs under the constraint of tangent vector across boundaries and achieved automated restoration of fragmented artifacts. Huang et al. [6] used spatial surface-matching techniques in a study of automated assembly of artifact fragments. Wang [7] developed an improved Hausdorff distance-matching algorithm for assembling fragmented artifacts, and applied this algorithm in virtual artifact restoration. Li [8] proposed an algorithm for the restoration of broken rigid bodies based on the matching of fracture surfaces, and showed that this method could be used for accurate assembly of relatively complex artifact fragments for restoration. Geoffrey et al. [9] achieved automated assembly of thin artifact fragments under the condition that the geometry of the artifact was unknown. This method is of important reference value to the virtual restoration of hollow artifacts, such as ceramic containers, bowls, and vases. However, all the above methods only apply to situations where all fragments of the broken artifacts are available and substantially preserved, and it is still difficult to perform virtual restoration of artifacts with missing parts.

In order to recover artifacts with missing parts in damaged areas, some scholars have proposed methods to estimate the geometry of the missing parts based on the symmetry of the artifact, in order to ensure the reliability of virtual restoration results. Li et al. [10] proposed a geometric method that estimates the symmetry axis of broken artifacts to obtain information on the generatrix of the artifact fragments, and applied this method to successfully restore an artifact model with inner and outer surfaces. Yang et al. [11] discussed the framework of a computer-aided artifact restoration system. They used quadratic surface fitting to calculate the normal vectors, and applied straight-line geometric methods to calculate the rotary axis of the entire object, in order to improve the precision of the calculation on the rotary axis. With this method, they successfully restored artifact fragments in the shape of common rotating bodies. Willis et al. [12] performed recombination on ceramic fragments with axial symmetry, and applied this method in the restoration of artifacts that show the property of axial symmetry. However, the application of the above methods is limited to broken artifacts with axial symmetry, such as bowls and pots. For artifacts in which the missing parts do not have a regular geometry, it is still challenging to obtain evidence for restoration.

Among the studies of virtual restoration of damaged artifacts with complex geometric configurations, researchers have used the constraints on local geometric features of the surface of the artifact model to create a smooth repair of missing parts [13-15]. In order to conduct virtual restoration of artifacts with relatively abundant geometric texture on the surface, Henning et al. [16] proposed an interactive cut-and-paste method based on multiresolution subdivision surfaces for texture restoration of three-dimensional artifact models. Yu et al. [17] used Poisson's equation as the theoretical basis to provide mesh editing, deformation, consolidation, and smoothing operations of artifact models. Wei [18] applied carving on the surface of the artifact model, as well as cutting and pasting the geometric texture and other operations to repair the damaged area of the artifact model. However, although these methods achieve free editing and operations on the surface of the artifact's model so that the artifact could be restored to a shape that met the restorer's expectations, it could not be reliably repaired by this method. In response to this, one research institute recombined the fragments of a terracotta statue damaged in a 2009 earthquake and used the geometric shape of the right hand of the statue as a reference for virtual restoration of the missing left hand, followed by exploring the restoration of its polychrome decoration (2013). Lu et al. [19] and Lu et al. [20] applied a method based on the matching of rigid surfaces, and performed a virtual restoration of damaged 
Buddha faces in artifact restoration and protection of the Bayon Temple in Cambodia, utilizing the characteristic that the four faces in the same spire bear similarities. Wu et al. [21] conducted virtual restoration on damaged hands of the rock carving of the Dazu Thousand-Hand statue, but their study mainly focused on damage to the surface area of the three-dimensional artifact modeler for incomplete statue hands that have missing parts, and these studies found no reliable evidence for the restoration.

\section{Methodology}

\subsection{Overall Process of the Proposed Method}

This research aims to achieve accurate and objective virtual restoration of damaged cultural relics with complex geometry. To solve the problem of virtual restoration to diversify the results, the proposed method was used to analyze the spatial geometric features of 3D cultural relic models under multiscales. That analysis can obtain historical information on the geometric shapes and sizes of the missing parts. On this basis, the result can show the historical origin of the relics. The whole process of virtual repair is shown in Figure 2.

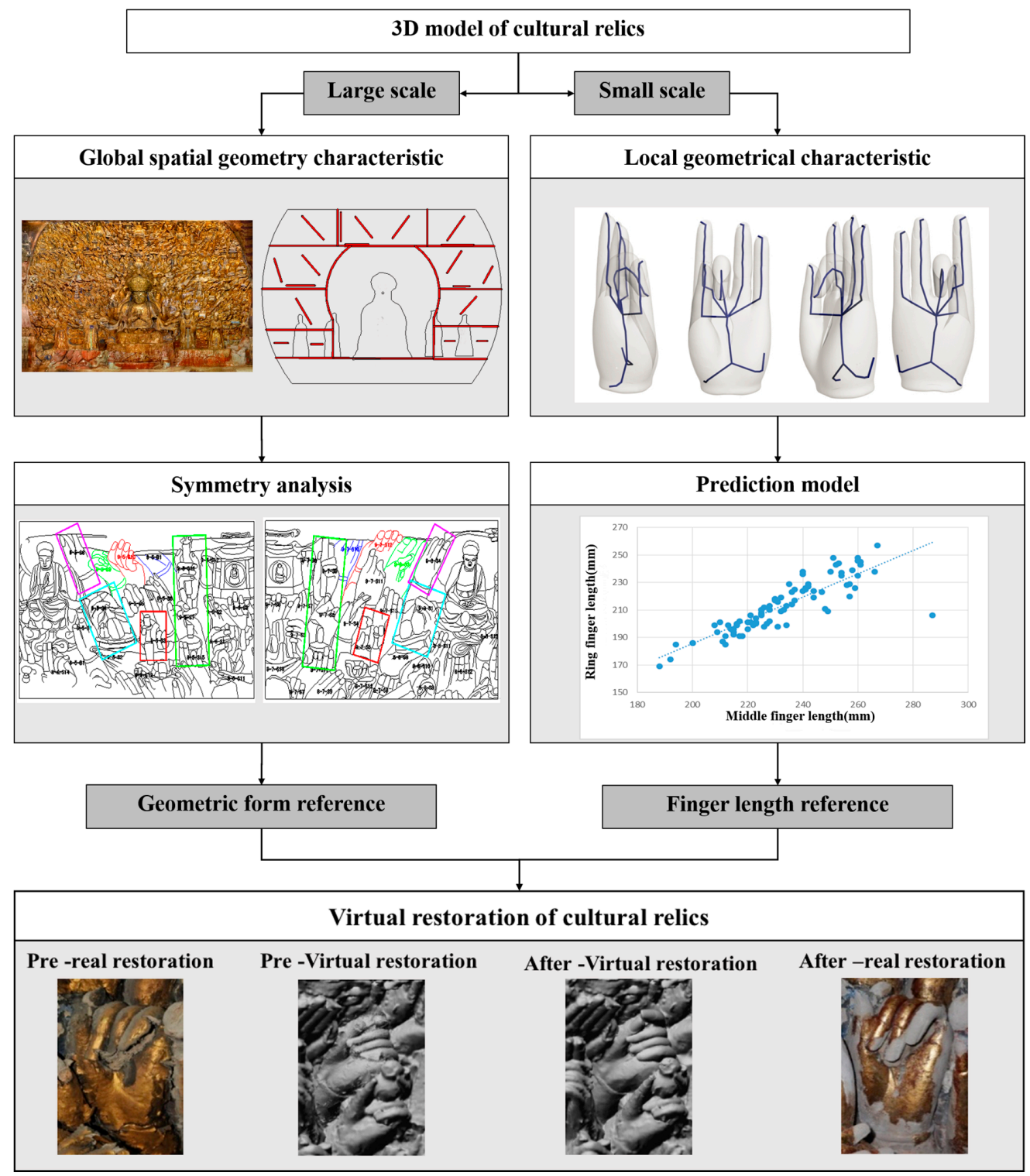

Figure 2. Overall technical process of the proposed method. 
The overall process is divided into two aspects: the first is to analyze the global geometric characteristics of the cultural relics on a large-scale model, and the second is to analyze the local geometric features on fine models with small scale. In the global space geometry analysis, the purpose is to find the geometric information of the defective part in the relic, then analyze the symmetry of the whole object. Using this method, the geometric reference samples of the defect object are usually obtained.

Further, in order to solve the condition of similar geometric shape but different proportion, the local geometric features of the cultural relic needs to be analyzed. This study uses the skeleton line of fine model to express the local geometric characteristics, because the skeleton line can express the geometric shape of each finger and its topologic relation while ignoring unwanted model surface noise. Through the statistics and analysis of artifacts in geometric characteristics, especially the skeleton line, the goal of local space geometry analysis is to establish a prediction model of the size of missing parts in relics. It is important to note that the limitations of the predictive model lie in the fact that artifacts are required to be grouped together or that subregions are grouped in a whole artifact.

Through these steps, the geometric shape and actual size of the missing part can be found. These are the constraints of virtual restoration of cultural relics. It is important that the constraint conditions are analyzed by the geometric characteristics of the relics so that the constraint conditions are in accordance with the history. After that, 3D reconstruction technology is used for virtual repair.

\subsection{Global Spatial Geometry Characteristics Analysis}

The analysis of global spatial geometry characteristics is mainly divided into two steps, spatial geometry, and similarity analysis. The geometric construction of cultural relics divides the relics into several subregions through a certain kind of spacing mechanism. The purpose of the geometric feature analysis of the entire cultural relic space is to examine the premise of whether there is any connection between geometries shown in relics. If this assumption holds, the inherent relationships between geometric constructs can be used to support the virtual restoration of artifacts. For example, after the cultural relics are divided into regions, they may exhibit spatial symmetry. In this way, the geometric similarity between structures can be utilized to obtain geometric references of artifacts. Geometric analysis of the space can vary according to the geometry of different artifacts, but the main considerations include the overall size of the artifacts, the overall axis of symmetry, and the center of the whole artifact.

\subsection{Local Geometric Characteristics Analysis}

\subsubsection{Extraction of Skeleton Line}

The local geometric features of cultural relics can usually be calculated by geometric calculation on the 3D model, but simple calculation cannot get accurate results on the skeleton line. Furthermore, the skeleton line of a 3D model of a cultural relic refers to the one-dimensional representation of the three-dimensional model geometry and its topologic structure. The three-dimensional model of complex geometric structure is expressed by the skeleton line, which not only reflects its local geometric features, but also preserves its topologic relation. The 3D model of the relic is expressed in this form, which makes it easier to analyze the local geometric features. Therefore, the skeleton line is of great significance to the virtual restoration of cultural relics.

For the extraction of skeleton lines of 3D models, the method based on the Laplace operator, proposed by $\mathrm{Au}$ [22], is adopted and improved by taking into account the topology and centrality of the skeleton lines.

The algorithm is divided into three steps: geometric contraction, skeleton simplification, and node fine-tuning. 
1. The geometric contraction process can be expressed as follows:

Step 1: Solve $\left[\begin{array}{cc}W_{L}^{t} & L^{t} \\ W_{H}^{t} & 0\end{array}\right] V^{t+1}=\left[\begin{array}{cc}0 & 0 \\ W_{H}^{t} & V^{t}\end{array}\right]$ and obtain the solution $V^{t+1}$, where $L$ is an $n \times n$ weighted Laplace operator of curvature flow, $V$ is a vertex of the manifold, and $W_{L}^{t}$ and $W_{L}^{t}$ are both $n \times n$ weighted diagonal matrices. These two matrices are used to balance the contraction strength and constraint of shape preservation during the iterative contraction process. The $i$ th element in the diagonal matrix $W_{L}^{t}\left(W_{H}^{t}\right)$ is defined as $W_{L, i}^{t}\left(W_{H, i}^{t}\right)$.

Step 2: Use the newly obtained solution $V^{t+1}$ to update $L^{t+1}$.

Step 3: Update the contraction weight and the constraint weight. $A$ is the area of the first-ring triangle mesh with $i$ as the vertex in the triangulation model, and $S_{L}$ is the contraction step from the origin to the $L$ vertex, where

$$
\begin{gathered}
W_{L}^{t+1}=S_{L} W_{L}^{t} \\
W_{H, i}^{t+1}=W_{H, i}^{0} \sqrt{A_{i}^{0} / A_{i}^{t}}
\end{gathered}
$$

Step 4: The condition for terminating the iteration is reached when the ratio of the model volume after contraction and the original model volume is less than $1 \times 10^{-5}$. If the termination condition is not reached, go back to Step 1; otherwise, the iteration is terminated.

2. Skeleton simplification is a method for further converting this mesh model of approximately zero volume to one-dimensional skeleton lines.

In this process, we define a quadratic error matrix $K_{i j}$ for each edge $(i, j)$ in the model (see Equation (3)). A matrix method based on the distance between the midpoint and the line of space can be obtained. The quadratic error measure of point $\mathrm{p}$ to its opposite side is $P^{T}\left(K_{i j}^{T} K_{i j}\right) P$.

$$
K_{i j}=\left[\begin{array}{cccc}
0 & -a_{z} & -a_{y} & -b_{x} \\
a_{z} & 0 & -a_{x} & -b_{y} \\
-a_{y} & a_{x} & 0 & -b_{z}
\end{array}\right]
$$

where $a$ is the normalized edge vector of edge $(i, j)$, and $b=a \times \hat{\mathrm{V}}_{i}$.

As shown in Equation (4), the initial quadratic error of vertex $i$ is the sum of squares of the distance from the point to its associated edge

$$
F_{i} \mathrm{p}=P^{T} \sum_{(i, j) \in E}\left(K_{i j}^{T} K_{i j}\right) P
$$

In the process of simplification, in order to guarantee the shape of the skeleton line as much as possible, the quadratic error measure produced by folding an edge $(i, j)$ is

$$
\varepsilon_{a}(i, j)=F_{i} \widetilde{\left(V_{j}\right)}+\widetilde{\left.V_{j}\right)}
$$

In this simplification algorithm, the method based on the quadratic error may easily cause oversimplification of the skeleton nodes when the geometry of the finger is close to a straight line. Hence, to ensure that the skeleton nodes with relatively even density on fingers with different curvatures in the same statue hand are obtained, the weights to appropriately amplify the quadratic errors in the model where the finger is approximately a straight line are added.

$$
\varepsilon_{b}(i, j)=\sum_{(i, k) \in E}^{n}\left\|\widetilde{V}_{i}-\widetilde{V}_{j}\right\|
$$


Thus, the overall quadratic error is the weighted sum of the above two parts of quadratic errors

$$
\varepsilon(i, j)=a \varepsilon_{a}(i . j)+b \varepsilon_{b}(i, j)
$$

3. In the node fine-tuning process, the generated skeleton nodes are divided into three categories: regular nodes, branch nodes, and terminal nodes. Assuming the upper and lower boundaries of the region is set and each boundary contains a vertex set $S_{j}$ that includes vertices in the original model, the weighted average displacement of the vertex $d_{j}$ in the boundary can be expressed by Equation (8)

$$
d_{j=} \frac{\sum_{i \in \operatorname{set}(j)} l_{i, j}\left(\widetilde{v}_{i}-v_{j}\right)}{\sum_{i \in \operatorname{set}(j)} l_{j, i}}
$$

where $l_{j, i}$ is the sum of the two adjacent sides converging to vertex $i$ in the first ring boundary $j$.

For ordinary nodes, there are only two adjacent edges in the skeleton point. Since the skeleton nodes correspond to the two ring boundaries $d_{1}$ and $d_{2}$ in the original model, the spatial coordinates of the skeleton points are adjusted to

$$
\mathrm{V}=\mathrm{V}-\frac{d_{1}+d_{2}}{2} \mathrm{~V}=\mathrm{V}-\frac{d_{2}+d_{2}}{2}
$$

where $\mathrm{V}$ is the spatial coordinates of the skeleton point.

For branch nodes that have three or more adjacent edges, in order to make the skeleton line of the model more in line with the topological relations among the geometric structures of the model ontology, it is necessary to merge the branch nodes that are too close. It should be noted that for models with different geometric constructions, the thresholds for branch node merging need to be adjusted according to the actual situation. Moreover, the centrality of the skeleton point of the branch node is adjusted to

$$
\mathrm{V}=\frac{d_{1} d_{2}+\ldots d_{n}}{N}
$$

The last kind of skeleton nodes are called end nodes, which are characterized by one point with only one adjacent edge. The fine-tuning method for this class of vertices is

$$
\mathrm{V}=\mathrm{V}-d \mathrm{~V}=\mathrm{V}-\mathrm{d}
$$

\subsubsection{Prediction Model}

In this study, regression analysis is used to fit the prediction model, which is used to calculate the length of broken relics (in this study, the finger length), to obtain reliable references for the restoration. The equation of the model is

$$
\mathrm{Y}=\hat{a}+\hat{b} x
$$

where $\hat{b}=\frac{n \sum_{i=1}^{n} x_{i} y_{i}-\left(\sum_{i=1}^{n} x_{i}\right)\left(\sum_{i=1}^{n} y_{i}\right)}{n \sum_{i=1}^{n} x_{i}^{2}-\left(\sum_{i=1}^{n} x_{i}\right)^{2}}, \hat{a}=\frac{1}{n} \sum_{i=1}^{n} y_{i}-\frac{\hat{b}}{n} \sum_{i=1}^{n} x_{i}, x$ and $y$ represent the lengths of two fingers on the same hand, $i$ is the $i$ th sample, and $n$ is the number of samples.

\section{Results and Analysis}

\subsection{Experimental Artifact}

To test the proposed method, the Dazu Thousand-Hand Bodhisattva statue in China was chosen as the study artifact, as shown in Figure 3a. This artifact is located in section no. 8 of the South Cliff of the Giant Buddha Bay in Baodingshan, Dazu District, Chongqing City. The Thousand-Hand statue was carved in the Southern Song Dynasty, dating back over 800 years. It is one of the most exquisite parts of the Dazu rock carvings. The Dazu Thousand-Hand Bodhisattva statue is famous for having nearly 1000 hands in different forms and holding different ritual instruments, positioned in a radial 
arrangement. The entire statue is covered with gold foil, showing a magnificent appearance, vivid forms, and resplendent gold color. However, due to its long history and environmental factors, damage such as rock weathering and peeling of the gold foil have occurred (Figure 3b). Because of this damage, many fingers in the hands are completely or partially missing, so that the rock statue can no longer faithfully represent its historical appearance, seriously affecting archaeological research. Hence, using three-dimensional laser scanning to digitize the geometry of the statue and perform virtual restoration of the incomplete fingers, aiming to precisely restore the statue's historical appearance, is important to both the protection of this artifact and archaeological research.

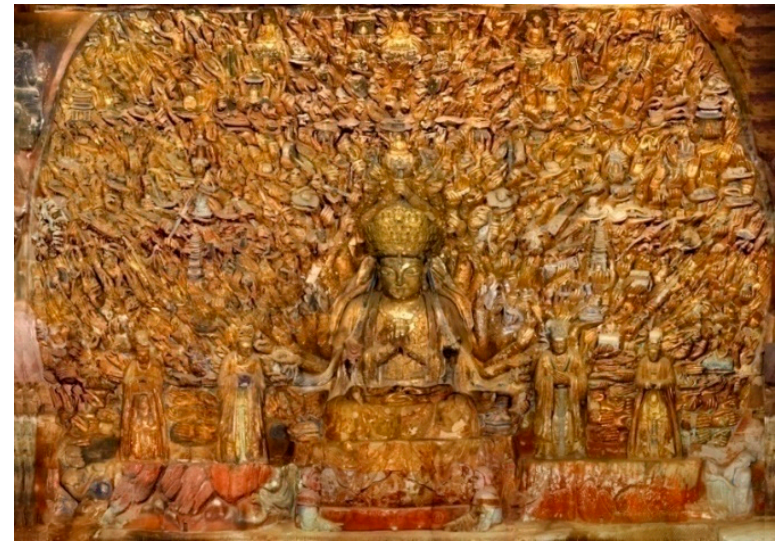

(a)

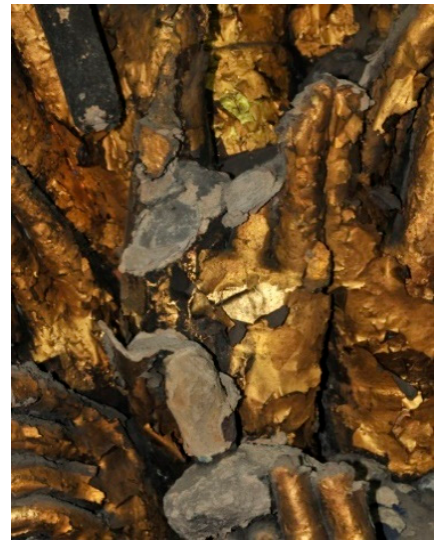

(b)

Figure 3. Dazu Thousand-Hand Bodhisattva statue: (a) orthophoto map; (b) one damaged hand.

\subsection{Experimental Data}

The data used in our research included different scales of three-dimensional point clouds. The three-dimensional point clouds of the whole shrine of the Dazu Thousand-Hand Bodhisattva statue were acquired using a FARO LS420 scanner (Figure 4a) with high measurement accuracy (position 3-6 mm, sampling density $1 \mathrm{~mm}$ ). These data were mainly used to construct a triangular mesh model of the whole niche. During the construction process, the original point cloud data needed to be preprocessed, including point cloud denoising and station. Because of the large size of the Guanyin statue, multistation data were required to be able to scan it intact. The complete model was formed by splicing the repeated parts between the stations. Finally, after repairing some holes caused by disease or occlusion of cultural relics, the three-dimensional model was built by the point cloud model encapsulation as shown in Figure 5a.

A CimCore Infinite 2.0 articulating arm (Figure $4 \mathrm{~b}$ ) was used to obtain finer scanning data (position $0.045 \mathrm{~mm}$, sampling density $0.1 \mathrm{~mm}$ ) of the hands and Dharma instruments by doing meticulous scanning one by one. These data were used to construct a high-precision triangular mesh model of the statue hands. These data show the detailed geometry of the Guanyin hands and the geometric texture of the surface. The difference with the above process is that after preprocessing the point cloud data, some redundant data caused by disease on the model surface, as the gold foil is missing from the surface, needed to be deleted. 


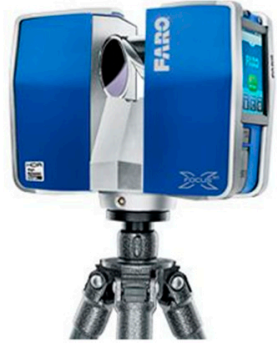

(a)

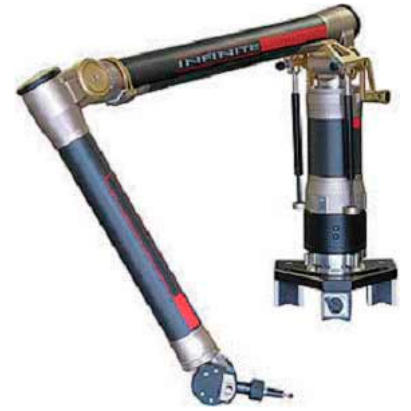

(b)

Figure 4. Equipment used in data acquisition: (a) FARO LS420 scanner; (b) CimCore Infinite 2.0 articulating arm.

\subsection{Global Spatial Geometry Characteristics Analysis}

\subsubsection{Spatial Partition}

The purpose of partitioning the Thousand-Hand Bodhisattva statue was to facilitate spatial analysis. According to the partitioning, each hand was given a unique identification. In the experiment, the whole statue was divided into a nine-by-nine grid, ending up with 81 areas covering the whole area. The lower left corner was the origin. The serial number of each hand was a combination of line number, column number, and number of hands in the area (Figure 6a). For example, the ID 9-7-s3 represents the third hand from the spectator at row 9, column 7 (Figure 6b).

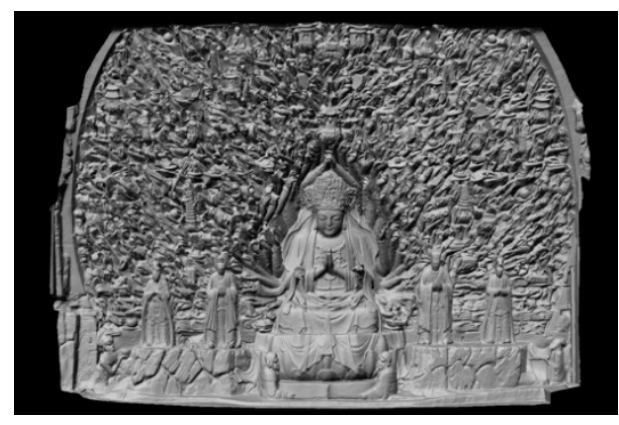

(a)

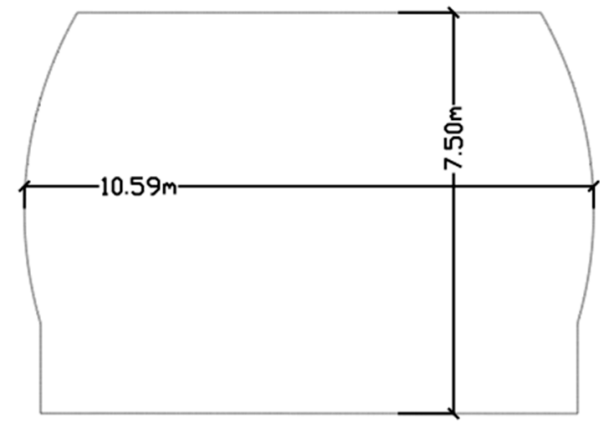

(b)

Figure 5. Geometric information of the Dazu Thousand-Hand Bodhisattva statue: (a) orthophoto quad; and (b) dimensions of statue model.

\subsubsection{Spatial Geometry Analysis}

It was important to determine the center point of the Dazu Thousand-Hand Bodhisattva statue to analyze the spatial distribution of the hands. Through three-dimensional measurement and statistics on the triangular mesh models that were reconstructed by point clouds, the results showed that the statue is about $7.5 \mathrm{~m}$ high and $10.59 \mathrm{~m}$ wide (Figure 5). Furthermore, there are 830 hands, of which 599 are relatively well maintained. 


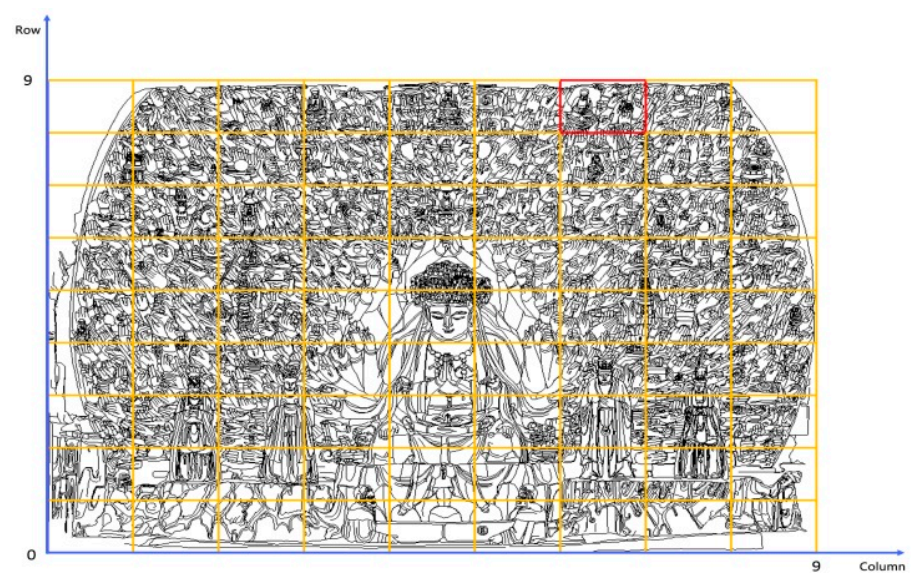

(a)

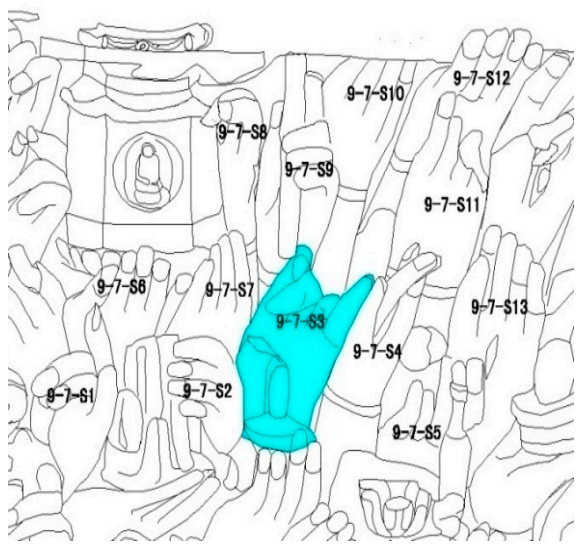

(b)

Figure 6. Spatial partitioning of the Dazu Thousand-Hand Bodhisattva statue: (a) partitioning; (b) line drawing of hand number 9-7-s3.

In the three-dimensional model shown in Figure 5a, the curved boundary on both sides of the statue can fit into an approximate ellipse. The basic parameters of the ellipse are as follows: long axis (vertical direction) $12.5 \mathrm{~m}$, short axis (horizontal direction) $10.59 \mathrm{~m}$, and flat curvature 0.85 . The center of the ellipse is measured by the origin. The objects are measured at the center of the ellipse. The result is that the upper boundary of the center is $3.70 \mathrm{~m}$, the lower boundary of the statue is $3.80 \mathrm{~m}$, and the boundary between the left and right sides is $5.30 \mathrm{~m}$ (see Figure 7a). In addition, the center of the ellipse is basically coincident with an extremely important and very special place called the "sky eye" on the Dazu Thousand-Hand Bodhisattva statue. Figure $7 \mathrm{~b}$ shows the positions of the center and "sky eye". In this study, the "sky eye" is considered to be the center of the whole statue. It is very important to analyze the symmetry of cultural relics. Through this position, the cultural relic can be divided vertically into left and right halves. On the two sides of the vertical bisector, the spectator can appreciate the characteristics of spatial symmetry and similar geometric morphology. 


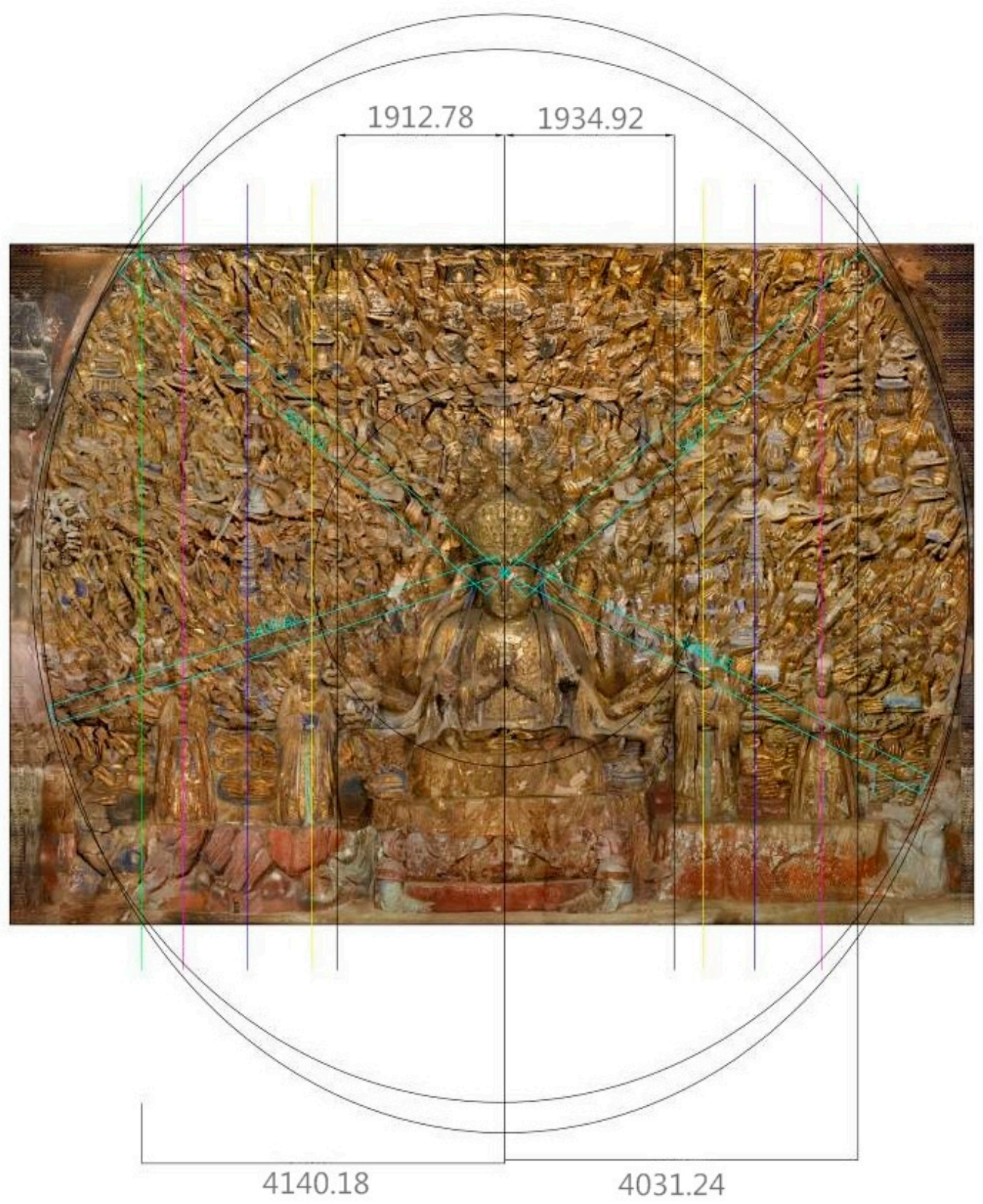

(a)

Figure 7. Cont. 


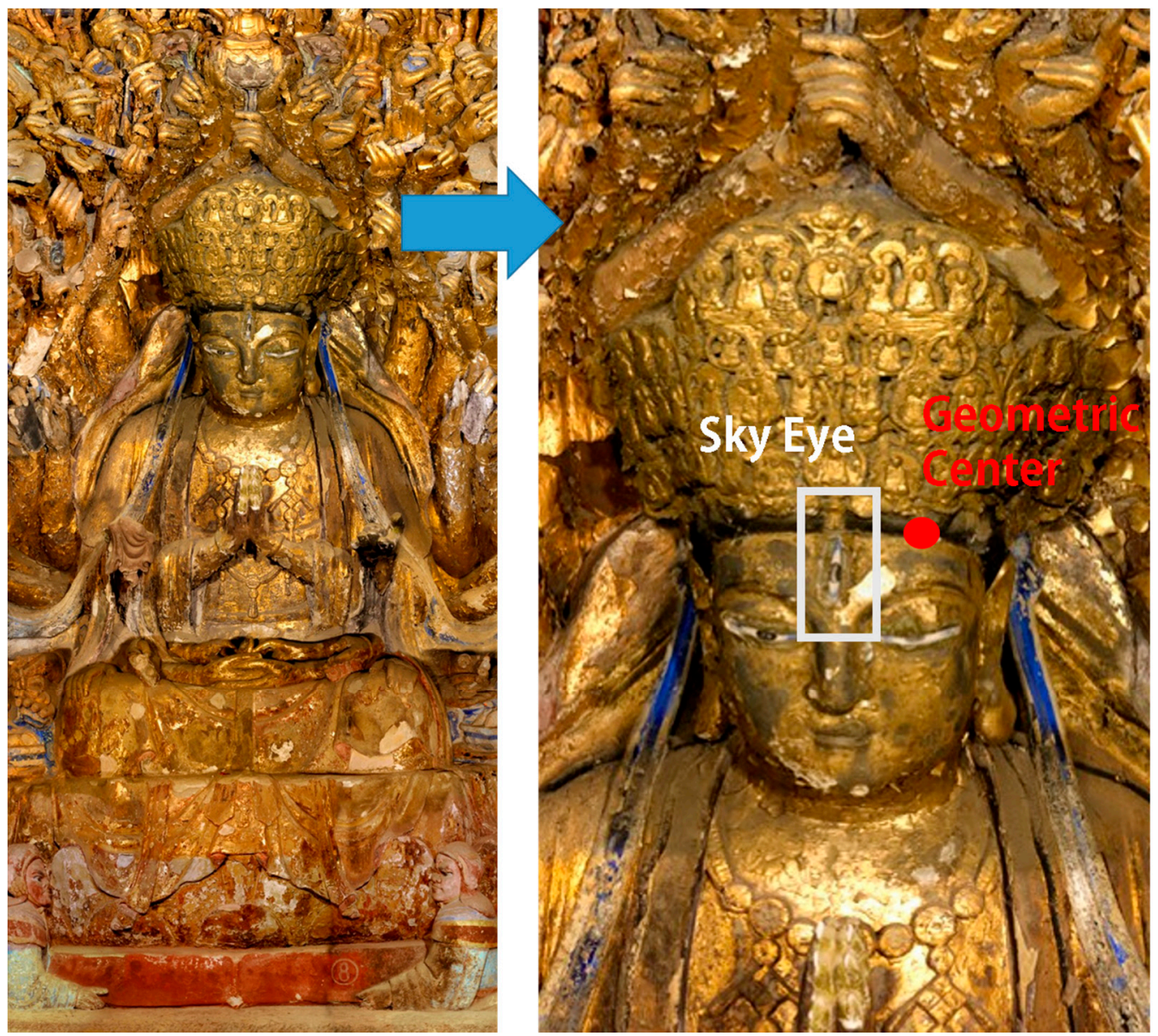

(b)

Figure 7. Global spatial geometric characteristics analysis of the Dazu Thousand-Hand Bodhisattva statue: (a) example of spatial geometric feature fitting on the object; (b) positions of geometric center point and "sky eye".

\subsubsection{Analysis of Global Geometric Similarity}

The purpose of analyzing the symmetries of the Dazu Thousand-Hand Bodhisattva statue is to find similar hands in the statue. The approximate geometric shape of a damaged hand can be obtained through similarities detected by the spectator. This study identifies similar hands through global geometric features and related religious knowledge, mainly including the following:

1. Symmetry of hand positions, whether the positions of both hands are based on the centerline symmetry of the statue.

2. Geometrical morphology, the main factor that determines whether the similarity of the geometric form of a symmetrical hand is the geometric form of each finger. Usually, each hand represents a gesture, which has a special religious meaning. Therefore, it is possible to check whether symmetrical hands express the same gesture. Finally, it can be judged by whether the hands hold the same instrument.

3. Neighboring relations, whether the hand adjacent to the target hand is symmetrical and similar in shape.

Based on the above principles, two corresponding hands can be found as the left hand and the right hand. If one of them is well preserved, it can provide a geometric reference to the corresponding broken one. 


\subsection{Analysis of Local Geometric Characteristics}

\subsubsection{Extraction of Skeleton Line}

The method for extracting skeleton lines was applied to the statue. After several experimental rounds, the iteration parameters for the three-dimensional model of the statue were obtained as follows: contraction strength $W_{L}^{0}=77.56$, contraction step $S_{0}=2.0$, shape preservation constraint $W_{H}^{0}=1.0$.

Figure 8 shows the process of the iterative contraction of the triangulation model of this statue hand. Each iteration is, in essence, one mesh contraction of the model. The shape preservation constraint of each vertex was then updated to ensure that the model contraction continues while preserving the key geometric details on the surface.

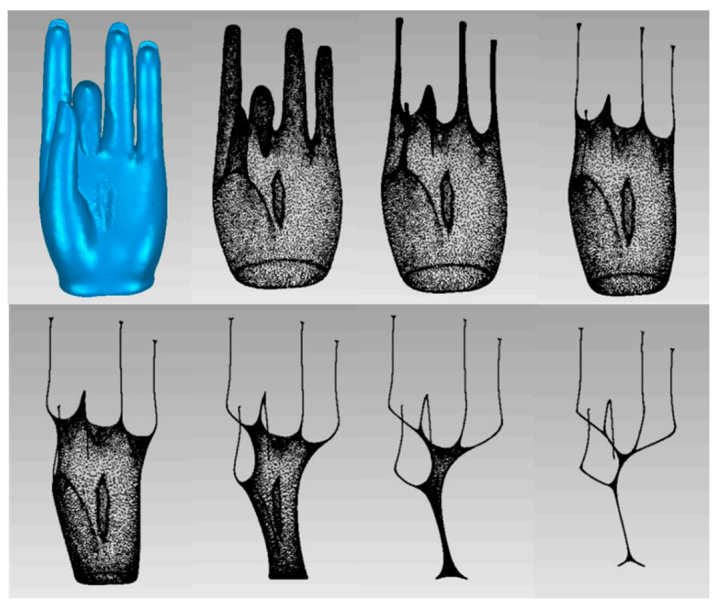

Figure 8. Iteration contraction process of cultural relic model.

According to Equations (3)-(7), when $a=1$ and $b=0.1,130$ skeleton nodes can be obtained, as shown in Figure 9a; when $a=1$ and $b=1,149$ skeleton nodes can be obtained, as shown in Figure $9 \mathrm{~b}$. Under the condition that the fingers are curved in the original hand model, adding weights does not have a large impact on the number of skeleton nodes. However, in areas where the geometric shape of the finger is relatively straight, adding weights increases the density of the skeleton nodes.

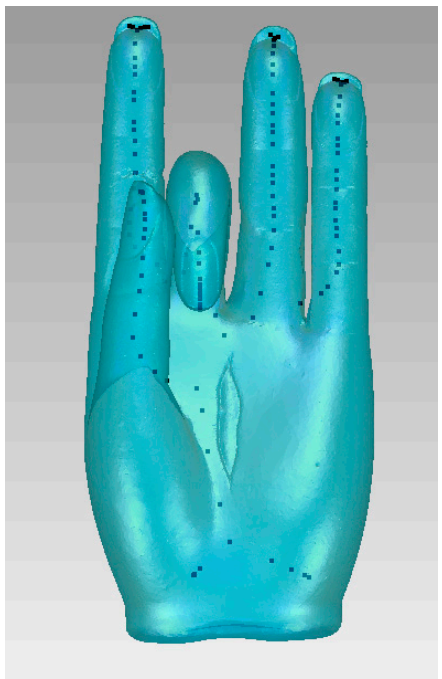

(a)

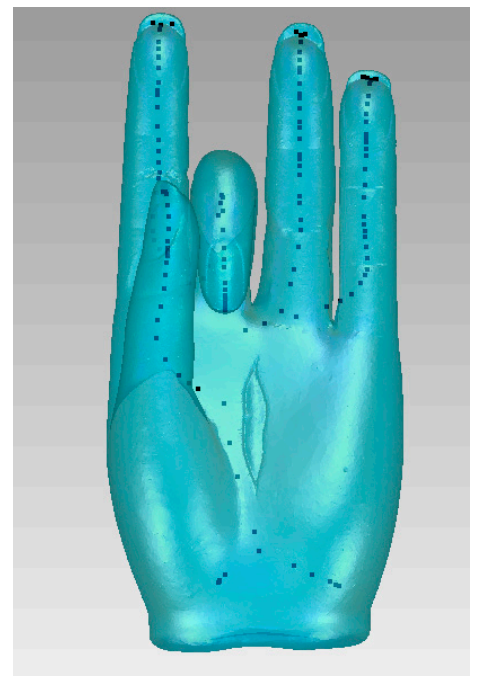

(b)

Figure 9. Simplification result of QEM (Quadric error metrics) skeleton nodes under different weights: (a) $a=1$, and $b=0.1,130$ skeleton nodes; (b) $a=1$ and $b=1,149$ skeleton nodes. 
Figure 10 shows the results of node fine-tuning. In view of the characteristics of the three-dimensional model of the hand in the statue, the topologic relation and the separation of some skeletons from the surface of the model are improved. The red dots and yellow lines are key skeleton nodes and lines, respectively, before taking into consideration tuning with centrality. The white dots and blue lines are the key skeleton nodes and lines, respectively, after the fine-tuning. As can be seen from the figure, after applying the fine-tuning algorithm that takes centrality into account, the key skeleton nodes at the thumb junction and between the little finger and the palm are moved to the correct positions. In addition, after the fine-tuning, the topologic relationships between the skeleton lines are more in line with the biological structure of human hands. Figure 11 illustrates the multiangle display of the skeleton lines extracted from the three-dimensional model using the skeleton line extraction algorithm that takes centrality into account.

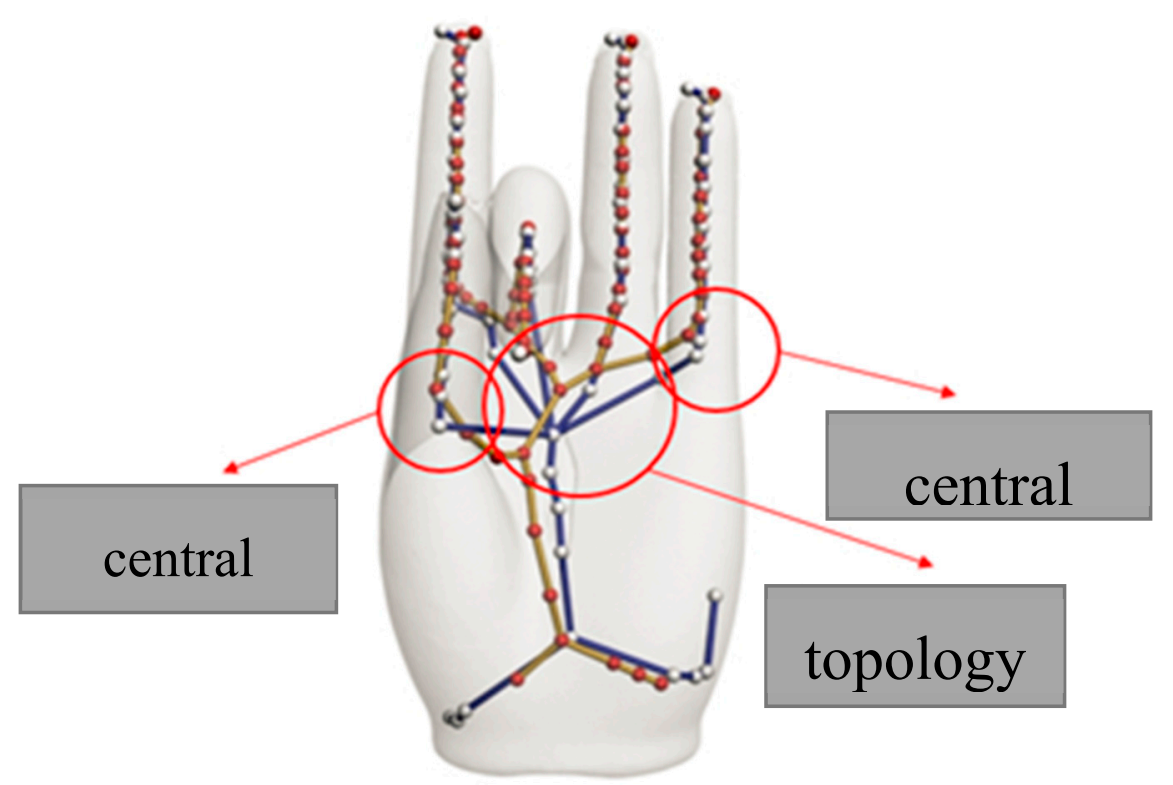

Figure 10. Comparison of skeleton nodes before and after fine-tuning.
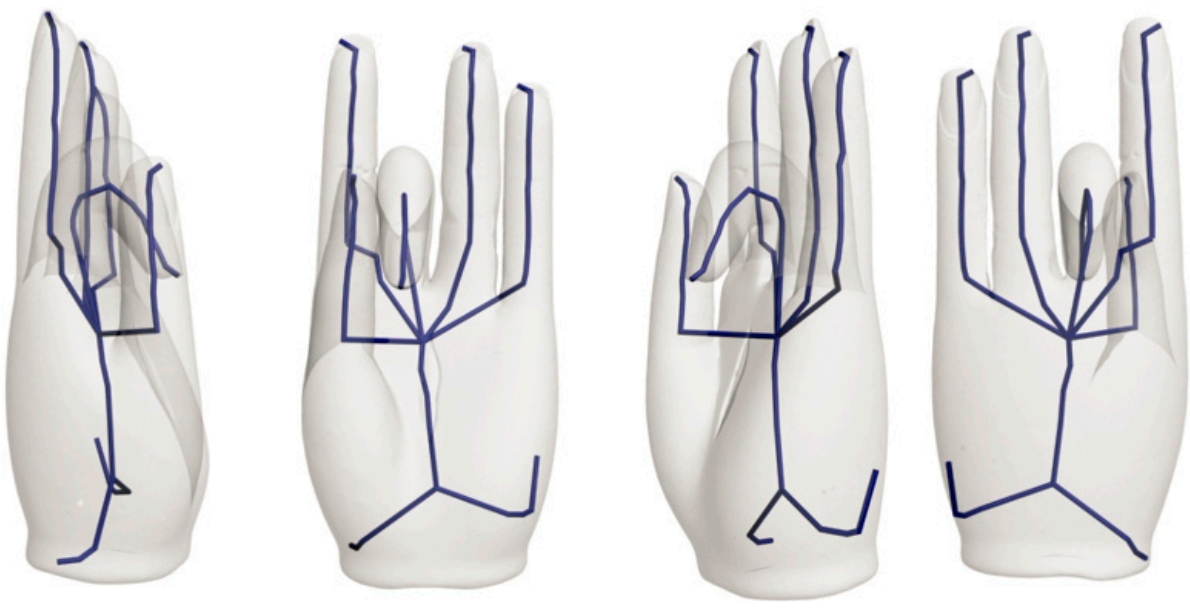

Figure 11. Multiangle displays of skeleton lines.

\subsubsection{Prediction of Finger Length}

Through the skeleton line extraction algorithm, the lengths of the skeletons of fingers indicate the real lengths of the fingers. Then, calculated finger lengths of the well-preserved hands are classified by 
thumb, forefinger, middle finger, ring finger, and little finger. Finally, the regression analysis method can be used to construct a mathematical model, which is a computational model of mathematical relations between various fingers. For the same hand, using the prediction model, the length of the broken finger can be estimated.

In the experiment, 66 well-preserved hands were identified on the Dazu Thousand-Hand Bodhisattva statue. Taking the index and middle fingers as an example to extract the length of the skeleton line, regression analysis was performed to check whether there was a linear relationship between different fingers.

\subsubsection{Hypothesis Testing and Anomaly Detection}

As shown in Figure 12a, a linear relationship can be observed. However, it is also obvious that there is a small number of outlier points (red dots). These unusual points may be caused by the hand grip, or by the natural form of the stone wall. Therefore, in order to ensure the accuracy of the proposed model, the anomaly points need to be excluded. In this paper, three statistical indices are used to detect abnormal points: centered leverage value, studentized deleted residual, and Cook's distance. Among them, the first two indices mainly detect a single finger length anomaly, the experimental selection centered leverage value is less than 0.03 , and the studentized deleted residual is between $(-1,1)$. The final index is used to detect the imbalance of the finger ratio, which is less than 0.01 . After the anomaly detection, six sets of data were excluded, shown by red dots in Figure 12b.

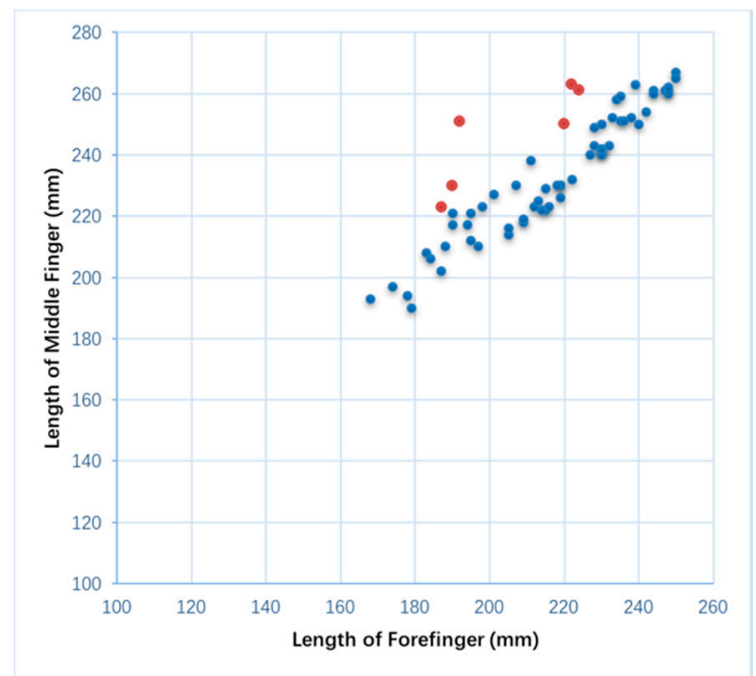

(a)

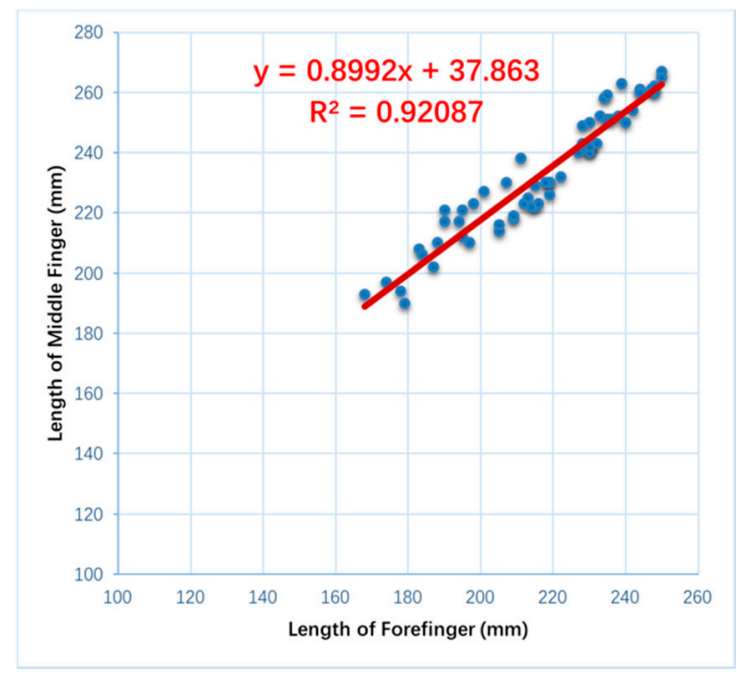

(b)

Figure 12. Prediction model: (a) hypothesis testing and outlier analysis of the data; (b) prediction model of incomplete finger length.

\subsubsection{Model Fitting}

After the hypothesis testing and outlier point exclusion, the lengths of the index fingers and middle fingers of 60 hands were fitted to the prediction model (Figure 12). The prediction model is

$$
\mathrm{Y}=0.8992 \mathrm{x}+37.863
$$

where $\mathrm{x}$ is the length of the index finger and $\mathrm{Y}$ is the length of the middle finger.

In this model, $\mathrm{R}^{2}=0.92087$, and $t$ test $p$ value is 0.02 , indicating that the prediction model has significant statistical significance. Two standard deviations were the length of the prediction interval, about $6.23 \mathrm{~mm}$. This length can be fine-tuned as an aesthetic. 


\subsection{Example of Virtual Restoration of Incomplete Hands}

This section describes virtual restoration of the hand ID 9-7-s4 (Figure 13a) on the Bodhisattva statue as an example. In this hand, the index finger is broken, and the middle finger, ring finger, and small finger have undergone weathering.

First, a similar hand, ID 9-5-s6 (Figure 13b), was found by analyzing the spatial geometry. Hands ID 9-7-s4 (broken finger) and ID 9-5-s6 (basically intact fingers) are geometrically similar, and their spatial location is symmetric based on the statue's center (Figure 14a). Furthermore, the hands adjacent to those two hands are also similar (Figure 14b). Therefore, the geometric shape of the missing parts of hand 9-7-s4 can refer to hand 9-5-s6.

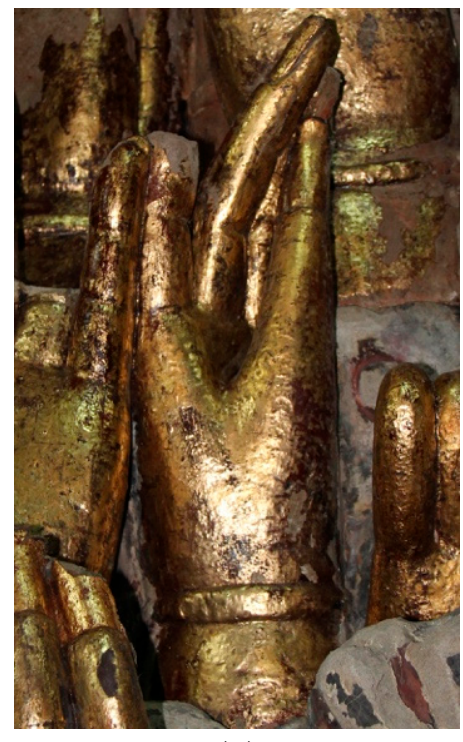

(a)

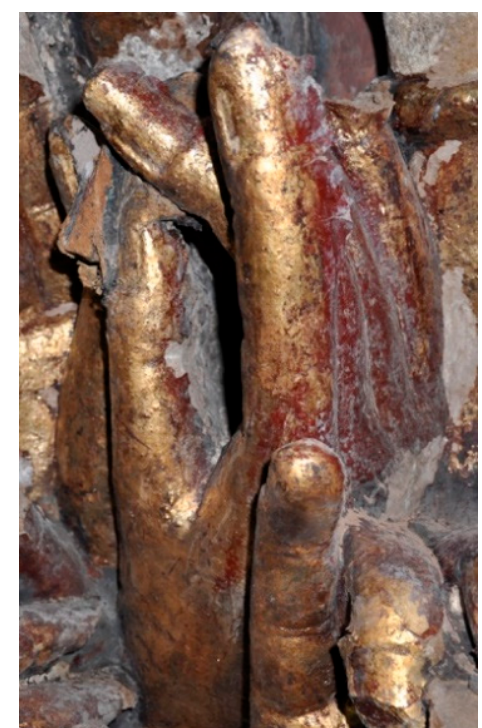

(b)

Figure 13. Photographs of missing fingers and their reference hands: (a) hand 9-7-s4; (b) hand 9-5-s6; which is the hand symmetric to 9-7-s4.

Second, the skeleton lines of the fingers in hand ID 9-7-s4 were extracted. Among them, the length of the middle finger is $241 \mathrm{~mm}$, and the length of the current index finger (broken) is $110 \mathrm{~mm}$. Through Equation (13), it can be concluded that the length of the broken index finger should be $226 \mathrm{~mm}$. Therefore, the finger length needs to be increased by $116 \mathrm{~mm}$ based on the geometric form of the index finger of 9-5-s6.

Finally, based on the geometric shape of the reference hand and the length of the finger calculated by the prediction model, the broken hand is repaired in a computer simulation. Geomagic 2017 commercial software was used to virtually recover the model by human-computer interaction. Figure 15 shows the results of this virtual restoration. 

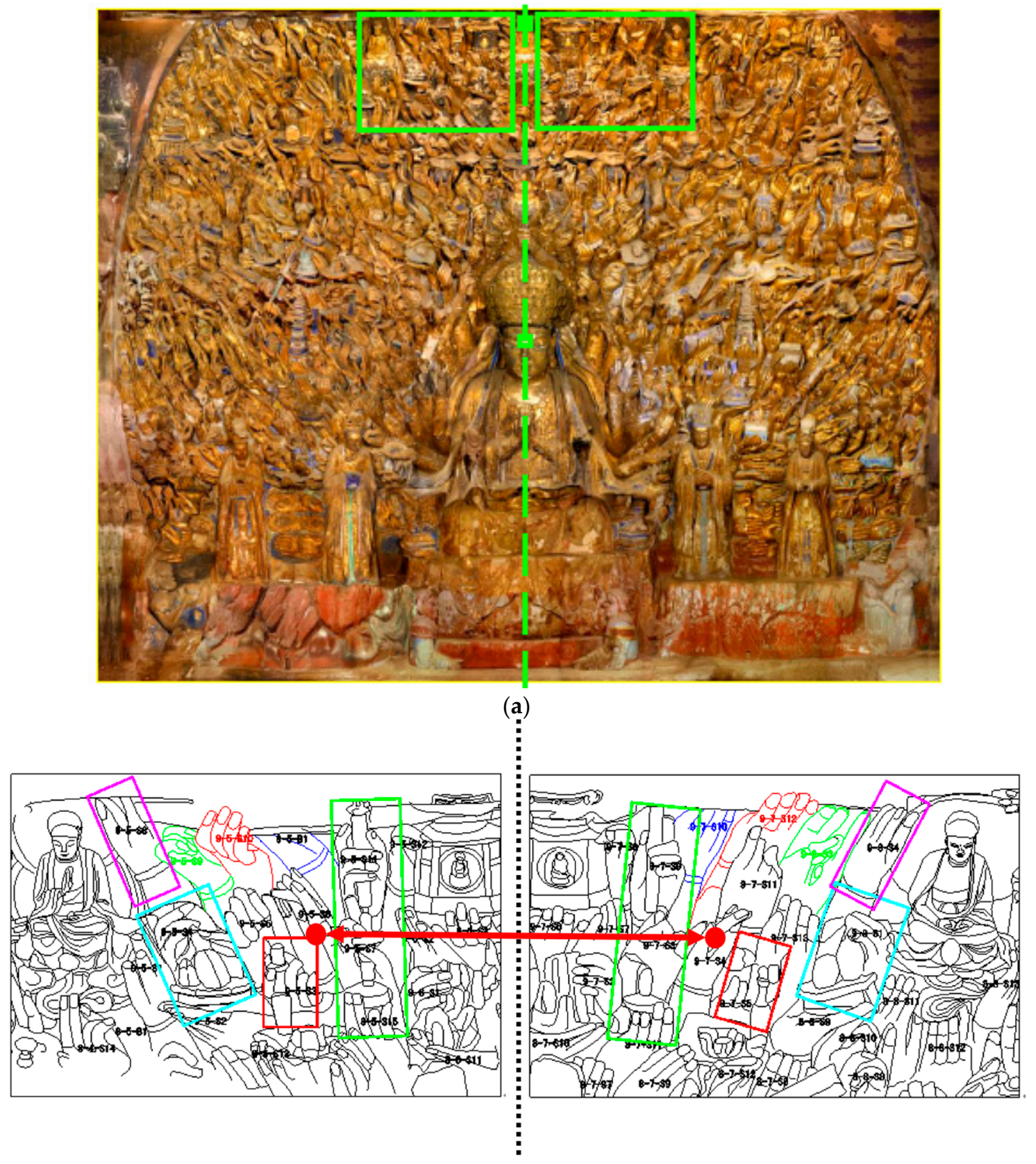

(b)

Figure 14. Characteristics of symmetry in the Dazu Thousand-Hand Bodhisattva statue: (a) space areas of hands 9-7-s4 (right side) and 9-5-s6 (left side); (b) local enlargement of line drawing with the geometrically similar hand.

There are altogether 231 incomplete Guanyin hands. Some of them can be repaired directly because of their few incomplete parts and obvious geometric shape. According to the above virtual repair process, a total of 58 broken fingers were repaired. 


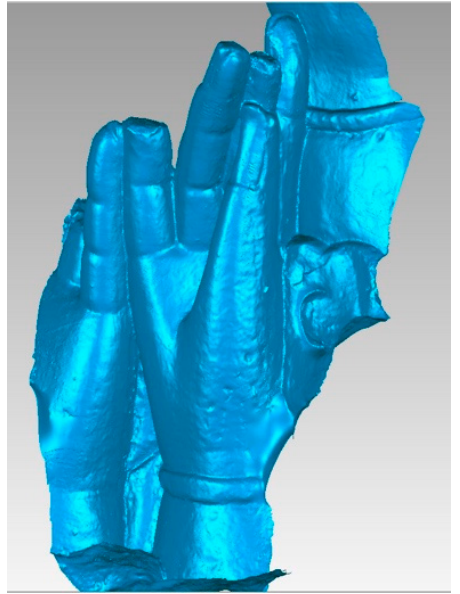

(a)

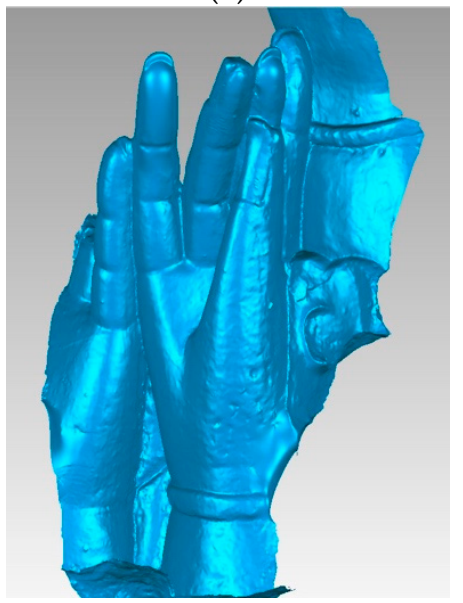

(c)

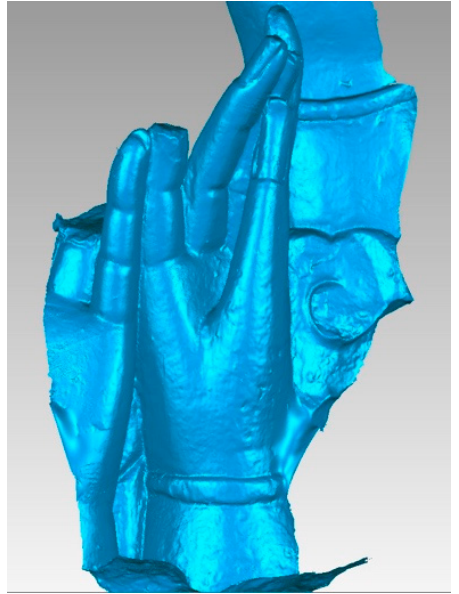

(b)

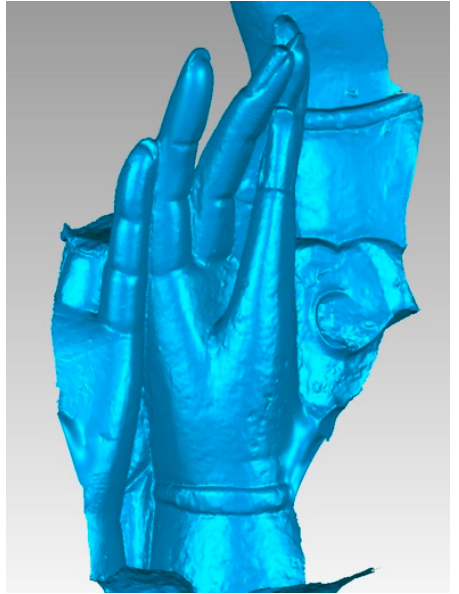

(d)

Figure 15. Virtual restoration effect of ID 9-7-s4 hand: (a,b) original model; $(\mathbf{c}, \mathbf{d})$ virtual restoration effect.

\section{Discussion}

In the virtual restoration of cultural heritage relics, the unknown geometries of damaged parts of artifacts present a huge challenge. How to make the virtual repair effect close to the historical appearance is a difficult problem in the absence of relevant historical records. This research used the Dazu Thousand-Hand Bodhisattva statue, part of China's world heritage, for the study. The virtual restoration effects were successfully applied to the conservation project of this cultural relic. The restoration workers refer to the virtual restoration effect provided by this method.

The method proposed in this paper not only can be used for virtual restoration of the Dazu Thousand-Hand Bodhisattva statue, but also can be applied to many other cultural relics. This is because many artifacts were designed and measured, not randomly constructed. Therefore, in most cases, cultural relics are regulated. If the tacit knowledge can be extracted by the geometric features of the relics and analyzed, this information can be used to recreate their historical shape and also provide a reference for the actual repair work. Therefore, if a cultural relic has a certain spatial distribution, or if there is a regression relationship between the different geometric parts, it can be repaired with the methods and ideas proposed in this paper. It is important to remember that the global geometric features are not limited to the cultural relic space symmetry, and the skeleton line is only one of the typical features of local geometry. In practical applications, according to the characteristics of cultural relics, different spatial geometry characteristics can be selected at different scales. Through the process of spatial and mathematical analysis, the implicit regulations of the spatial geometry of cultural relics can be discovered. At the same time, virtual restoration based on the geometric 
features of different scales can complement and verify each other. This also has great significance for archaeology. In summary, this method has strong reference and repeatability potential in the field of cultural relic restoration.

In future work, researchers could consider creating a 3D semantic database of the hands' 3D models and richly describe each one-such as geometric position, shape of gesture, etc.-in order to achieve semantics-based spatial analysis and retrieval. The advantage of this method is that on the basis of a geometric morphologic analysis of cultural relics, it can also use semantics to retrieve the symmetry and similarity of cultural relics, and it is easier to analyze the degree of matching.

\section{Conclusions}

This paper presents a practical method for virtual restoration of artifacts with complex geometric structures based on multiscale spatial geometry, especially those artifacts that have missing and broken parts and no proper documented information. In this paper, for the first time, the geometric shape and size of cultural relics are determined by analyzing the global and local geometric features. Based on this, objective virtual restoration of cultural relics is carried out. In the repair process, the spatial symmetry of relics is utilized. The skeleton line was first proposed to express the three-dimensional models. The adaptive adjustment of the skeleton line extraction algorithm improves the central and topological relationship of the skeleton line. In the end, the length prediction model of the relic is established by regression analysis.

This method mainly solves the problem that the complex geometry of relics is difficult to find in the evidence of virtual restoration and is more scientific and objective than subjective judgment. The effect of virtual restoration can be explained by the results of multiscale spatial geometric analysis of cultural relics. The results of this experiment have been successfully applied to the virtual restoration of the Dazu Thousand-Hand Bodhisattva stone carvings. In addition, the methods and ideas provided in this paper also apply to the virtual restoration of other cultural relics, which has great reference value. Therefore, the method is of great significance to the virtual restoration of complex geometric structures.

Author Contributions: Conceptualization, M.H. and S.Y.; Methodology, Y.H. and Y.W.; Software, L.J., S.Z., and P.W.; Validation, S.Y.; Formal analysis, M.H. and S.Y.; Investigation, S.Z. and P.W.; Resources, M.H. and Y.H.; Data curation, L.J.; Writing-Original draft preparation and review, all authors.

Funding: The research work conducted in this paper was supported by the research fund of the National Key Research and Development Program (no. 2016YFB0501404 and no. 2017YFB1402525) and the Beijing Advanced Innovation Center for Future Urban Design (no. UDC2016030200), and by Supporting Plan for Cultivating High Level Teachers in Colleges and Universities in Beijing (no. IDHT20170508).

Acknowledgments: The authors would like to acknowledge and thank the Dazu Museum for its support of this work. The authors also would like to thank Ian Dowman from University College London, UK, and Songnian Li from Ryerson University, Canada, for their valuable comments on improving the manuscript, Changfa Zhan from Dazu Rock Carvings Museum in Chongqing for his novel suggestions, and Fangyin Li and Huili Chen from Dazu Rock Carvings Museum in Chongqing for their work on the capturing and processing of the data.

Conflicts of Interest: The authors declare no conflict of interest.

\section{References}

1. Su, Y.Q. A Comprehensive Study on Three-Dimensional Digital Restoration of Qingzhou Buddha Statues. Master's Thesis, Yantai University, Yantai, China, 2008.

2. Yang, L.B. The Application of Shape Matching Technology in Artifact Restoration. Master's Thesis, Northwest University, Xi'an, China, 2002.

3. Ru, S.F. A Study on the Restoration of Fragmented Objects and Computer-Aided Artifact Restoration. Ph.D. Thesis, Northwest University, Xi'an, China, 2004.

4. Pan, R.J. Several Problems in Computer-Aided Artifact Restoration. Ph.D. Thesis, Henan University of Science and Technology, Luoyang, China, 2005.

5. Fan, S.R. Methods for Shaping Matching and Assembly of Complementary Parts of Rigid Bodies. Master's Thesis, Northwest University, Xi'an, China, 2005. 
6. Huang, Q.X.; Flöry, S.; Gelfand, N.; Hofer, M.; Pottmann, H. Reassembling fractured objects by geometric matching. ACM Trans. Graph. 2006, 25, 569-578. [CrossRef]

7. Wang, Y.T. The Application of Contour Matching Technology in Artifact Restoration. Master's Thesis, Henan University of Science and Technology, Luoyang, China, 2011.

8. Li, Q.H.; Zhou, M.Q.; Geng, G.H. Reassembly of broken 3D solids based on fractured surfaces matching. J. Image Graph. 2012, 17, 1298-1304.

9. Oxholm, G.; Nishino, K. A flexible approach to reassembling thin artifacts of unknown geometry. J. Cult. Herit. 2013, 14, 51-61. [CrossRef]

10. Li, C.L.; Zhou, M.Q.; Cheng, X.; Cheng, R.B. Virtual restoration of axisymmetric relic fragments. J. Comput.-Aided Des. Comput. Graph. 2006, 18, 620-624.

11. Yang, C.L.; Zhang, Z.X.; Pan, R.J. A study on the framework of computer-aided artifact repair system and key technologies. J. Syst. Simul. 2006, 18, 2003-2006.

12. Willis, A.R.; Cooper, D.B. Bayesian assembly of 3D axially symmetric shapes from fragments. In Proceedings of the 2004 IEEE Computer Society Conference on Computer Vision and Pattern Recognition (CVPR 2004), Washington, DC, USA, 27 June-2 July 2004; Volume 81, pp. I-82-I-89.

13. Chalmoviansky, P.; Juettler, B. Filling holes in point clouds. Lect. Notes Comput. Sci. 2003, 2768, $196-212$.

14. Nooruddin, F.S.; Turk, G. Simplification and repair of polygonal models using volumetric techniques. IEEE Trans. Vis. Comput. Graph. 2003, 9, 191-205. [CrossRef]

15. Ju, T. Robust repair of polygonal models. ACM Trans. Graph. 2004, 23, 888-895. [CrossRef]

16. Biermann, H.; Martin, I.; Bernardini, F.; Zorin, D. Cut-and-paste editing of multiresolution surfaces. ACM Trans. Graph. 2002, 21, 312-321. [CrossRef]

17. Yu, Y.Z.; Zhou, K.; Xu, D.; Shi, X.H.; Bao, H.J.; Guo, B.N.; Shum, H.Y. Mesh editing with poisson-based gradient field manipulation. ACM Trans. Graph. 2004, 23, 644-651. [CrossRef]

18. Wei, T. Data Integration and Interactive Repair Techniques for 3D Geometric Modeling. Master's Thesis, Peking University, Beijing, China, 2007.

19. Lu, M.; Kamakura, M.; Zheng, B.; Takamatsu, J.; Nishino, K.; Ikeuchi, K. Clustering bayon face towers using restored $3 \mathrm{D}$ shape models. In Proceedings of the Second International Conference on Culture and Computing, Kyoto, Japan, 20-22 October 2011; pp. 39-44.

20. Lu, M.; Zheng, B.; Takamatsu, J.; Nishino, K.; Ikeuchi, K. 3D shape restoration via matrix recovery. Lect. Notes Comput. Sci. 2010, 6469, 306-315.

21. Wu, Y.H.; Hou, M.L.; Zhang, Y.M. Progress and future direction of the application of 3D laser scanning technology in rock artifact protection. Geomat. World 2011, 4, 53-57.

22. Au, K.C.; Tai, C.L.; Chu, H.K.; Cohen-Or, D.; Lee, T.Y. Skeleton extraction by mesh contraction. ACM Trans. Graph. 2008, 27, 1-10. [CrossRef] 